\title{
Reliability Analysis Method Study of Multi-state System Based on Fuzzy Bayesian Network
}

\author{
Zhai Sheng ${ }^{*}$, , Shi Wuxi ${ }^{2}$ and Xiu Chunbo ${ }^{2}$ \\ ${ }^{I}$ School of Mechanical Engineering, Tianjin Polytechnic University, Tianjin, 300387, P.R. China \\ ${ }^{2}$ School of Electrical Engineering and Automation, Tianjin Polytechnic University, Tianjin, 300387, P.R. China
}

\begin{abstract}
To deal with fuzzy and uncertain information in reliability analysis of multi-state systems, an approach based on fuzzy Bayesian networks is proposed. In this approach the fuzzy set theory and Bayesian networks methodology are applied. The fuzzy assessment information from different experts is described by triangular fuzzy numbers, which denotes the fuzzy probabilities of different states of root nodes. The depended uncertain ordered weighted averaging (DUOWA) operator is used to aggregate assessments information. Through defuzzification calculation, the crisp probabilities are obtained as the prior probabilities of the root nodes. Bayesian network $(\mathrm{BN})$ is used to handle the uncertainty of logical relationship between nodes and to compute system reliability. A case study concerning the reliability analysis in cell production line is provided and conducted using the proposed approach to illustrate its effectivity and practicability.
\end{abstract}

Keywords: Bayesian networks, fuzzy number, multi-state system, reliability analysis.

\section{INTRODUCTION}

The traditional reliability theories including Reliability Block Diagram (RBD), Binary Decision Diagram (BDD), Fault Tree Analysis (FTA), etc. have been applied extensively in reliability analysis of systems [1-3]. Faced with increasingly complex systems and work environments, the requirements for systems reliability analysis are getting stringent, which makes the application of these traditional reliability theories restricted a lot [4]. The uncertainty and fuzziness are inherent to complex systems.

In recent years, originated in the field of artificial intelligence, Bayesian Network $(\mathrm{BN})$ as the representative of describing uncertain knowledge and advanced inference technology has been widely applied in reliability analysis $[5,6]$, risk analysis $[7,8]$ and fault diagnosis $[9,10]$. Due to its representation ability of multi-state characteristic and uncertainty of logical relationship between nodes, Mahadevan [11] and Wilson [12] established reliability analysis models of two-state and multi-state system using BN technique. And through BN bidirectional inference, the marginal probability and posterior probability were obtained to analyze the weaknesses in the system, which provided decision-making basis of design and maintenance of the system.

BN model above is based on the condition that the elementary events probabilities are the exact values, but in practical cases, due to the lack of historical data, systems and environments complexity, the exact values of the elementary events probabilities are difficult to obtain. To this end, in the risk forecast of subway fire Lu [13] used fuzzy numbers in fuzzy set theory to represent the fuzzy probabilities of elementary events, by defuzzification, the crisp values of fuzzy probabilities of elementary events were obtained, but failed to establish a reliability analysis modeling of a multi-state system. Chang et al. [14] considered both fuzzy states and fuzzy information. Simon [15] and Weber developed a Bayesian network model for determining reliability of the system with fuzzy states and imprecise probabilities.

In the process of synthesizing multiple expert judgments, $\mathrm{Lu}$ [13] integrated the multiple expert assessments using arithmetic average method, ignoring the different experts' weights. And in many cases, due to the policy-makers' limited expertise in related problem areas, it is difficult to specify the experts' weights, which makes experts' weights uncertain. And determining the experts' weights is mostly subjective, which may also cause a large deviation and affect the final evaluation.

A reliability analysis method of multi-state system based on fuzzy Bayesian networks is proposed to handle the fuzziness of data and uncertainty of logical relationship between events. The fuzzy uncertainty of the elementary events probabilities is described using fuzzy numbers in fuzzy set theory, the uncertainty of experts' weights is determined using depended uncertain ordered weighted averaging (DUOWA) [16] operator, the BN model is used to express the uncertain causal relationship between events and to compute system reliability.

The rest of this paper is organized as follows: Section 2 introduces the representation and integration of the experts' assessments. Section 3 briefly reviews the Bayesian network and introduces how to model multi-state system reliability based on BN. Section 4 illustrates the proposed approach 


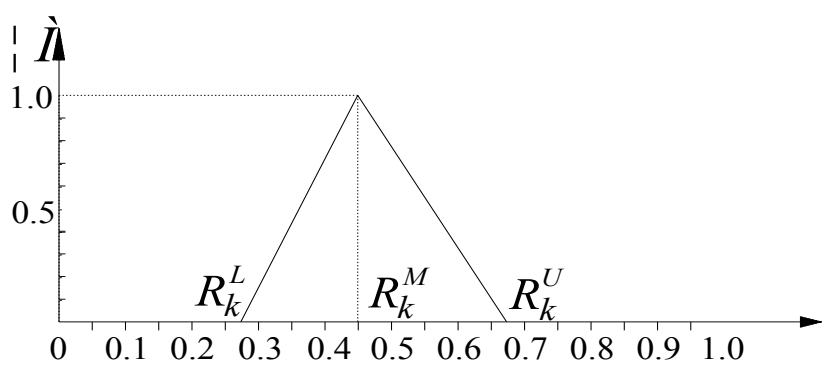

Fig. (1). Expert assessment in the form of triangular fuzzy number.

application in a cell production line. Section 5 draws the conclusion.

\section{REPRESENTATION AND AGGREGATION OF THE EXPERTS' ASSESSMENTS}

\subsection{Representation of the Experts' Assessments}

Under the conditions of being difficult to obtain exact events probabilities, the results are drawn from the experts' experience through the method of group decision-making. As the experts' assessments are often fuzzy information, in order to objectively describe the experts' assessments, in this paper the fuzzy numbers in fuzzy set theory is used to represent the experts' assessments, i.e. the fuzzy probabilities of events. Using fuzzy numbers to describe the occurrence probabilities of events may reduce the difficulty of obtaining the exact values.

There are many forms of fuzzy number, such as the interval fuzzy number, triangular fuzzy number, trapezoidal fuzzy number, normal fuzzy number and LR fuzzy number. This paper takes the form of triangular fuzzy number to represent the experts' assessments. The fuzzy probability may be expressed in the form of fuzzy set, shown in Fig. (1).

The triangular fuzzy number may be denoted as $\tilde{R}_{k}=$ $\left(R_{k}^{L}, R_{k}^{M}, R_{k}^{U}\right)$. The triangle membership function $\mu_{\tilde{R}}(x)$ is expressed as:

$\mu_{\tilde{R}}(x)=\left\{\begin{array}{c}\frac{x-R_{k}^{L}}{R_{k}^{M}-R_{k}^{L}}, \quad R_{k}^{L} \leq x \leq R_{k}^{M} \\ \frac{R_{k}^{U}-x}{R_{k}^{U}-R_{k}^{M}}, \quad R_{k}^{M} \leq x \leq R_{k}^{U} \\ 0, \quad \text { otherwise }\end{array}\right.$

\subsection{Aggregation of the Experts' Assessments}

In the case of being difficult to determine the experts' weights, in order to achieve the objective evaluation of the results, this paper relies on DUOWA operator to integrate the experts' assessments, that is, by calculating the similarity degree between individual values and the average assessed value to determine the experts' weights. The more similar it is, the bigger the weight is. The less similar it is, the smaller the weight is.
Suppose an expert panel composed of $n$ experts makes events probabilities assessments. The triangular fuzzy number of assessment value of $k$-th expert is $\tilde{R}_{k}=\left(R_{k}^{L}, R_{k}^{M}\right.$, $\left.R_{k}^{U}\right)$, the average value is $\tilde{R}_{a}=\left(R_{a}^{L}, R_{a}^{M}, R_{a}^{U}\right)$, and $R_{a}^{L}=\frac{1}{n} \sum_{k=1}^{n} R_{k}^{L}, \quad R_{a}^{M}=\frac{1}{n} \sum_{k=1}^{n} R_{k}^{M}, \quad R_{a}^{U}=\frac{1}{n} \sum_{k=1}^{n} R_{k}^{U}$,

$k=1,2, \cdots, n$.

The distance $d\left(\tilde{R}_{k}, \tilde{R}_{a}\right)$ between $\tilde{R}_{k}$ and $\tilde{R}_{a}$ is:

$d\left(\tilde{R}_{k}, \tilde{R}_{a}\right)=\frac{1}{3}\left(\left|R_{k}^{L}-R_{a}^{L}\right|+\left|R_{k}^{M}-R_{a}^{M}\right|+\left|R_{k}^{U}-R_{a}^{U}\right|\right)$

The similarity degree $s\left(R_{k}, R_{a}\right)$ between $\tilde{R}_{k}$ and $\tilde{R}_{a}$ is:

$s\left(\tilde{R}_{k}, \tilde{R}_{a}\right)=1-\frac{d\left(\tilde{R}_{k}, \tilde{R}_{a}\right)}{\sum_{k=1}^{n} d\left(\tilde{R}_{k}, \tilde{R}_{a}\right)}$

Using DUOWA operators to integrate the experts' assessments, the group assessment value $\tilde{R}$ is obtained as:

$\tilde{R}=\left(R^{L}, R^{M}, R^{U}\right)=\operatorname{DUOWA}\left(\tilde{R}_{1}, \tilde{R}_{2}, \ldots, \tilde{R}_{n}\right)$

$=\sum_{k=1}^{n} \frac{s\left(\tilde{R}_{k}, \tilde{R}_{a}\right) \times \tilde{R}_{k}}{\sum_{k=1}^{n} s\left(\tilde{R}_{k}, \tilde{R}_{a}\right)}$

Using the mean area method to defuzzify, the group assessment value $\tilde{R}$ is transformed into the crisp group assessment value $R$. The defuzzification formula of the mean area method is:

$R=\frac{R^{L}+2 R^{M}+R^{U}}{4}$

\section{MULTI-STATE SYSTEMS RELIABILITY MODEL- ING BASED ON BN}

\subsection{Bayesian Network}

$\mathrm{BN}$ is a Directed Acyclic Graph (DAG) of statistical dependence between random variables, which can be expressed as $B=\{G, P\}=\{X, E, P\}$, where $G$ is a DAG representing variable domain, $P$ represents a group of the corresponding 
set of conditional probabilities, $X$ is a random variable set, $X=\left\{x_{1}, x_{2}, \ldots, x_{l}\right\}$, each node in $G$ represents a random variable, and each node corresponds to a conditional probability table (CPT), $E$ is the set of directed edges, each edge in $G$ represents the dependency between variables. Each node $x_{i}$ in a BN predetermined graph is independent of any child nodes consisted of $x_{i}$ non descendant nodes given by $x_{i}$ parent node.

If $A\left(x_{i}\right)$ represents a subset of any node consisted of nondescendant nodes, indicating the direct parent node of $x_{i}$ with $\pi\left(x_{i}\right)$, then according to the conditional independence assumptions, there is:

$P\left(x_{i} \mid A\left(x_{i}\right), \pi\left(x_{i}\right)\right)=P\left(x_{i} \mid \pi\left(x_{i}\right)\right)$

Assuming $x_{1}, x_{2} \ldots x_{l}$ are the nodes of $\mathrm{BN}$, the joint probability of all nodes in the network as follows:

$$
P\left(x_{1}, x_{2}, \ldots, x_{l}\right)=\prod_{i=1}^{k} P\left(x_{i} \mid \pi\left(x_{i}\right)\right)
$$

So, BN can express joint probability distribution of the variables, and greatly simplify the solving of joint probability of variables.

\subsection{Multi-state Systems Reliability Modeling Based on BN}

Procedure for modeling multi-state system reliability based on $\mathrm{BN}$ is as follows:

1. Determining the nodes of BN, the root node of network representing the elementary events in the system, leaf nodes representing the system.

2. According to the causal relationship between the nodes determining the parent node, the child node, and establishing DAG which expresses the interdependence relationship between the nodes.

3. Determining the distribution parameters of priori probability of the root node and CPT.

In this paper, the $\mathrm{BN}$ model is established using method of Fault Tree (FT) mapping. FT model is established based on logical relationships between variables, and then the FT model is mapped into a $\mathrm{BN}$ model. A mapping algorithm includes graphical and numerical tasks. In graphical mapping, primary events, intermediate events, and the top event of the FT are represented as root nodes, intermediate nodes, and the leaf node in the corresponding $\mathrm{BN}$, respectively. In numerical mapping, the occurrence probabilities of the primary events are assigned to the corresponding root nodes as prior probabilities [11].

In BN model, the root node variable of BN is $\boldsymbol{x}_{\boldsymbol{i}}(\boldsymbol{i}=\mathbf{1 , 2}$, $\ldots, l)$, its state is $v_{i}\left(v_{i}=\mathbf{0}, \mathbf{1}, \ldots, r-1\right)$; intermediate node variable is $\mathbf{y}_{j}(j=1,2, \ldots, m)$, its state is $w_{j}\left(w_{j}=\mathbf{0}, \mathbf{1}, \ldots, s-1\right)$; the leaf node variable is $T$, and its state is $\boldsymbol{Q}_{T}\left(\boldsymbol{Q}_{T}=\mathbf{0}, \mathbf{1}, \ldots\right.$, $\boldsymbol{n - 1}$ ), Where in the normal state is state 0 . Given occurrence probability of various fault states of each root is $P\left(x_{i}=v_{i}\right)$, using the bucket elimination method, the occurrence probability $\boldsymbol{P}\left(\boldsymbol{T}=\boldsymbol{Q}_{T}\right)$ is obtained when the leaf node $\boldsymbol{T}=\boldsymbol{Q}_{\boldsymbol{T}}$ :

$$
\begin{aligned}
& P\left(T=Q_{T}\right) \\
& =\sum_{x_{1}, x_{2}, \mathrm{~L}, x_{l}, \mathrm{y}_{1}, y_{2}, \mathrm{~L}, \mathrm{y}_{m}} P\left(x_{1}, x_{2}, \mathrm{~L}, x_{l}, \mathrm{y}_{1}, \mathrm{y}_{2}, \mathrm{~L}, \mathrm{y}_{m}, T=Q_{T}\right) \\
& =\sum_{\pi(T)} P\left(T=Q_{T} \mid \pi(T)\right) \times \sum_{\pi(y 1)} P\left(y_{1} \mid \pi\left(y_{1}\right)\right) \cdots \times \sum_{\pi(y)} P\left(y_{j} \mid \pi\left(y_{j}\right)\right) \cdots \times \\
& \sum_{\pi(y m)} P\left(y_{m} \mid \pi\left(y_{m}\right)\right) P\left(x_{1}=v_{1}\right) \cdots P\left(x_{i}=v_{i}\right) \cdots P\left(x_{l}=v_{l}\right)
\end{aligned}
$$

When the root node $\boldsymbol{x}_{\boldsymbol{i}}=\boldsymbol{v}_{\boldsymbol{i}}$, the probability of leaf node

$$
\boldsymbol{T}=\boldsymbol{Q}_{\boldsymbol{T}} \text { is: }
$$

$P\left(T=Q_{T} \mid x_{i}=v_{i}\right)=\frac{P\left(T=Q_{T}, x_{i}=v_{i}\right)}{P\left(x_{i}=v_{i}\right)}$

$=\frac{\left.\sum_{x_{1}, x_{2}, \cdots, x_{l}, y_{1}, y_{2}, \cdots, y_{m}} \mathrm{y}_{m}, T=Q_{T}, x_{i}=v_{i}\right)}{P\left(x_{i}=v_{i}\right)}$

When a leaf node $\boldsymbol{T}=\boldsymbol{Q}_{\boldsymbol{T}}$, the state probability (posterior probability) of root node $\boldsymbol{x}_{\boldsymbol{i}}=\boldsymbol{v}_{\boldsymbol{i}}$ is:

$$
\begin{aligned}
& P\left(x_{i}=v_{i} \mid T=Q_{T}\right)=\frac{P\left(x_{i}=v_{i}, T=Q_{T}\right)}{P\left(T=Q_{T}\right)} \\
& =\frac{\sum_{x_{1}, x_{2}, \cdots, x_{i}, y_{1}, y_{2}, \cdots, y_{m}} P\left(x_{1}, x_{2}, \cdots, x_{l}, \mathrm{y}_{1}, \mathrm{y}_{2}, \cdots, \mathrm{y}_{m}, T=Q_{T}, x_{i}=v_{i}\right)}{P\left(T=Q_{T}\right)}
\end{aligned}
$$

According to Bayes' theorem:

$$
P\left(x_{i}=v_{i} \mid T=Q_{T}\right)=\frac{P\left(x_{i}=v_{i}\right) P\left(T=Q_{T} \mid x_{i}=v_{i}\right)}{P\left(T=Q_{T}\right)}
$$

In order for the reliability analysis of multi-state system, importance analysis is indispensable. Next, the state importance and the probability importance of the node will be defined.

Definition 1: When the root node $\boldsymbol{x}_{\boldsymbol{i}}$ is in the fault state $\boldsymbol{v}_{\boldsymbol{i}}$, the state importance $I_{Q_{T}}^{\mathrm{Pr}}\left(x_{i}=v_{i}\right)$ of the leaf node $\boldsymbol{T}=\boldsymbol{Q}_{\boldsymbol{T}}$ in this state is defined as:

$$
I_{Q_{T}}^{\mathrm{Pr}}\left(x_{i}=v_{i}\right)=P\left(T=Q_{T} \mid x_{i}=v_{i}\right)-P\left(T=Q_{T} \mid x_{i}=0\right)
$$

It represents the effective degree on the leaf nodes $\boldsymbol{T}=\boldsymbol{Q}_{\boldsymbol{T}}$ when root node $\boldsymbol{x}_{\boldsymbol{i}}=\boldsymbol{v}_{\boldsymbol{i}}$.

Definition 2: when the leaf node $T$ is in the state $\boldsymbol{Q}_{T}$, the probability importance $I_{Q_{T}}^{\mathrm{Pr}}\left(x_{i}\right)$ of root node $\boldsymbol{x}_{i}$ is defined as:

$$
\begin{aligned}
& I_{Q_{T}}^{\mathrm{Pr}}\left(x_{i}\right)=\frac{1}{r-1} \sum_{v_{i}=1}^{r-1} I_{Q_{T}}^{\mathrm{Pr}}\left(x_{i}=v_{i}\right) \\
& =\frac{1}{r-1} \sum_{v_{i}=1}^{r-1}\left[P\left(T=Q_{T} \mid x_{i}=v_{i}\right)-P\left(T=Q_{T} \mid x_{i}=0\right)\right]
\end{aligned}
$$




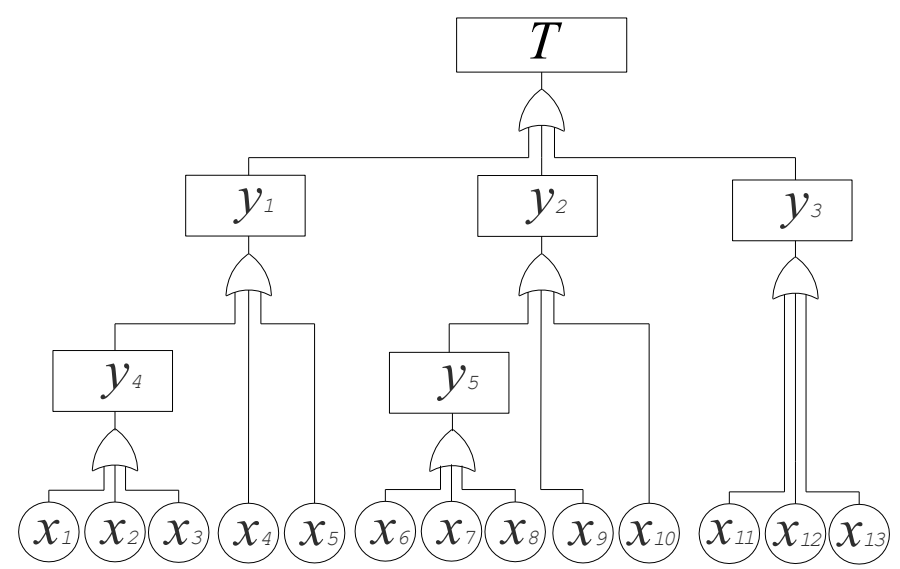

Fig. (2). Fault Tree Model of Feeding $\mathrm{MnO}_{2}$ Subsystem.

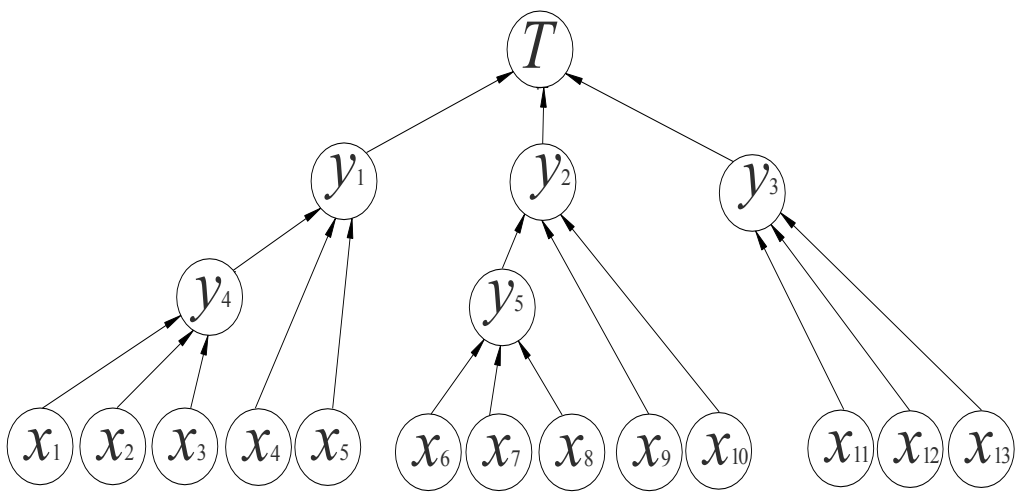

Fig. (3). BN model of subsystem feeding $\mathrm{MnO} 2$.

It represents the effective degree of the root node $x_{i}$ on the leaf node $\boldsymbol{T}=\boldsymbol{Q}_{\boldsymbol{T}}$.

Thus calculating the state importance and probability importance of each root node in the different fault states of system, we can know their impact on the system and find out the weak link of system. Calculating the posterior probabilities, we can identify the occurrence probability of each root node when the system is in fault state, and find out the cause of system fault. Finally, we provide reliable decision support for the design and maintenance of the system.

\section{CASE STUDY}

The following will be the reliability analysis of a cell production line for an enterprise. The main task of the production line is: putting the lithium-chip, the separator paper and the manganese cathode soaked by electrolyte into the negative electrode with set nets, and supplementing with volatile electrolyte in cathode film. Under the premise to ensure complete penetration of electrolyte, there are some other processes such as stamping cathode casing, sealing etc.

\subsection{Establishment of BN Reliability Modeling}

According to the function of the system, the system is divided into five working subsystems, namely the subsystem of feeding negative shell and lithium, subsystem of dividing the paper into negative shell, subsystem of feeding MnO2, subsystem of assembling positive steel shell, sealing subsystem. Among them the subsystem of feeding $\mathrm{MnO} 2$ is selected for analysis.

According to the logical relationship between events, FT model which top event is subsystem fault of feeding $\mathrm{MnO}_{2}$ is established, shown in Fig. (2).

Specification between the events and the notations is shown in Table $\mathbf{1}$.

The FT model being built is mapped to the BN model (omitted CPT due to space reason) as shown in Fig. (3), so the reliability model based on $\mathrm{BN}$ is established.

According to the practical situation, the components and the system are set in three states $0,1,2$, that is, $\boldsymbol{Q}_{T}=0,1,2$ and $\boldsymbol{v}_{\boldsymbol{i}}=0,1,2$. Where 0 is normal operation state, 1 is a state of slight fault state, 2 is serious fault state.

\subsection{Reliability Analysis System}

1) Experts' assessments.

First, a panel of 4 experts is established, according to their experience the experts assess the occurrence probability of each fault mode and the state, and then give the corresponding triangular fuzzy numbers. Using DUOWA operator 
Table 1. Nodes description.

\begin{tabular}{|c|c|c|c|}
\hline Node & Event & Node & Event \\
\hline \hline$T$ & Subsystem feeding MnO2 fault & $x_{5}$ & Sensor fault \\
\hline$y_{1}$ & Device feeding negative shell fault & $x_{6}$ & Cylinder feeding MnO2 jam \\
\hline$y_{2}$ & Device feeding MnO2 fault & $x_{7}$ & Defective MnO2 \\
\hline$y_{3}$ & Out-feeding device fault & $x_{8}$ & Solenoid valve fault \\
\hline$y_{4}$ & Cylinder feeding negative shell fault & $x_{9}$ & Conveyor belt fault \\
\hline$y_{5}$ & Cylinder feeding MnO2 fault & $x_{10}$ & Sensor fault \\
\hline$x_{1}$ & Cylinder feeding negative shell jam & $x_{11}$ & Solenoid valve fault \\
\hline$x_{2}$ & Negative shell deformation & $x_{12}$ & Out-feeding cylinder jam \\
\hline$x_{3}$ & Solenoid valve fault & $x_{13}$ & Sensor fault \\
\hline$x_{4}$ & Conveyor belt fault & & \\
\hline
\end{tabular}

Table 2. Expert assessment value and the exact value of the fault probability of the root node.

\begin{tabular}{|c|c|c|c|c|c|c|}
\hline Title & $\mathbf{x 1 = 1}$ & $\mathbf{x 2 = 1}$ & $\mathbf{( x 3}, \mathbf{x 8}, \mathbf{x 1 1})=\mathbf{2}$ & $\mathbf{x 4 = 1}$ & $\mathbf{x 4 = \mathbf { 2 }}$ & $\mathbf{( x 5}, \mathbf{x 1 0}, \mathbf{x 1 3})=\mathbf{1}$ \\
\hline \hline Expert 1 & $(0.15,0.22,0.3)$ & $(0.1,0.16,0.22)$ & $(0.38,0.43,0.52)$ & $(0.22,0.26,0.32)$ & $(0.1,0.15,0.2)$ & $(0.05,0.1,0.15)$ \\
\hline Expert 2 & $(0.18,0.25,0.32)$ & $(0.08,0.18,0.25)$ & $(0.35,0.45,0.55)$ & $(0.24,0.28,0.32)$ & $(0.08,0.16,0.22)$ & $(0.08,0.15,0.2)$ \\
\hline Expert 3 & $(0.2,0.25,0.3)$ & $(0.1,0.18,0.22)$ & $(0.4,0.45,0.5)$ & $(0.2,0.25,0.3)$ & $(0.09,0.14,0.19)$ & $(0.06,0.1,0.17)$ \\
\hline Expert 4 & $(0.16,0.2,0.25)$ & $(0.08,0.13,0.20)$ & $(0.37,0.42,0.47)$ & $(0.22,0.28,0.33)$ & $(0.08,0.13,0.18)$ & $(0.1,0.15,0.2)$ \\
\hline Group & $(0.173,0.231,0.295)$ & $(0.092,0.164,0.223)$ & $(0.377,0.437,0.509)$ & $(0.220,0.268,0.323)$ & $(0.089,0.145,0.197)$ & $(0.072,0.125,0.180)$ \\
\hline Exact value & 0.232 & 0.161 & 0.44 & 0.270 & 0.144 & 0.126 \\
\hline
\end{tabular}

(2-2)

\begin{tabular}{|c|c|c|c|c|c|}
\hline Title & $\mathbf{x 6 = 1}$ & $\mathbf{x 7 = 1}$ & $\mathbf{x 9 = 1}$ & $\mathbf{x 9 = 2}$ & $\mathbf{x 1 2 = 1}$ \\
\hline \hline Expert 1 & $(0.18,0.24,0.3)$ & $(0.12,0.18,0.24)$ & $(0.24,0.28,0.34)$ & $(0.1,0.15,0.2)$ & $(0.28,0.35,0.4)$ \\
\hline Expert 2 & $(0.2,0.25,0.3)$ & $(0.1,0.18,0.25)$ & $(0.26,0.3,0.34)$ & $(0.08,0.16,0.22)$ & $(0.25,0.3,0.35)$ \\
\hline Expert 3 & $(0.22,0.26,0.32)$ & $(0.1,0.15,0.22)$ & $(0.23,0.28,0.35)$ & $(0.09,0.14,0.19)$ & $(0.26,0.32,0.36)$ \\
\hline Expert 4 & $(0.2,0.26,0.3)$ & $(0.12,0.18,0.25)$ & $(0.24,0.3,0.25)$ & $(0.08,0.13,0.18)$ & $(0.27,0.33,0.38)$ \\
\hline Group & $(0.200,0.253,0.304)$ & $(0.111,0.174,0.241)$ & $(0.242,0.290,0.345)$ & $(0.089,0.145,0.197)$ & $(0.265,0.325,0.372)$ \\
\hline Exact value & 0.252 & 0.175 & 0.292 & 0.144 & 0.322 \\
\hline
\end{tabular}

method, according to Eq. (2-4) the estimation information of experts are integrated, the results of group assessment are obtained, after defuzzification by Eq. (5) the exact value of occurrence probability of each fault mode in different states is obtained, as shown in Table 2.

2) Calculation of the importance.
The exact values gained after defuzzification as priori probabilities are used to conduct inference calculation. Next we take node $x_{4}$ for example.

As the fault state of node $x_{4}$ is 1 , i.e. $v_{4}=1$, the state importance $I_{1}^{\mathrm{Pr}}\left(x_{4}=1\right)$ when $\boldsymbol{T}=\mathbf{1}$ can be obtained by Eq. (12) as follows: 
Table 3. State Importance of Nodes.

\begin{tabular}{|c|c|c|c|c|c|c|c|c|c|}
\hline \multirow{2}{*}{ Node } & \multicolumn{4}{|c|}{ State Importance } & \multirow{2}{*}{ Node } & \multicolumn{4}{|c|}{ State Importance } \\
\hline & $I_{1}^{\mathrm{Pr}}\left(x_{i}=1\right)$ & $I_{2}^{\mathrm{Pr}}\left(x_{i}=1\right)$ & $I_{1}^{\mathrm{Pr}}\left(x_{i}=2\right)$ & $I_{2}^{\mathrm{Pr}}\left(x_{i}=2\right)$ & & $I_{1}^{\mathrm{Pr}}\left(x_{i}=1\right)$ & $I_{2}^{\mathrm{Pr}}\left(x_{i}=1\right)$ & $I_{1}^{\mathrm{Pr}}\left(x_{i}=2\right)$ & $I_{2}^{\mathrm{Pr}}\left(x_{i}=2\right)$ \\
\hline$x_{1}$ & 0.99353 & 0 & 0 & 0 & $x_{8}$ & 0 & 0 & 0 & 0.99883 \\
\hline$x_{2}$ & 0.9949 & 0 & 0 & 0 & $x_{9}$ & 0.99374 & 0 & 0 & 0.99520 \\
\hline$x_{3}$ & 0 & 0 & 0 & 0.99883 & $x_{10}$ & 0.99343 & 0 & 0 & 0 \\
\hline$x_{4}$ & 0.99372 & 0 & 0 & 0.99854 & $x_{11}$ & 0 & 0 & 0 & 0.99883 \\
\hline$x_{5}$ & 0.99343 & 0 & 0 & 0 & $x_{12}$ & 0.99362 & 0 & 0 & 0 \\
\hline$x_{6}$ & 0.99355 & 0 & 0 & 0 & $x_{13}$ & 0.99343 & 0 & 0 & 0 \\
\hline$x_{7}$ & 0.99504 & 0 & 0 & 0 & & & & & \\
\hline
\end{tabular}

Table 4. Probability Importance of Nodes.

\begin{tabular}{|c|c|c|c|c|c|}
\hline \multirow{2}{*}{ Node } & \multicolumn{2}{|c|}{ Probability Importance } & \multirow{2}{*}{ Node } & \multicolumn{2}{|c|}{ Probability Importance } \\
\cline { 2 - 5 } & $I_{1}^{\mathrm{Pr}}\left(x_{i}\right)$ & $I_{2}^{\mathrm{Pr}}\left(x_{i}\right)$ & & $I_{1}^{\mathrm{Pr}}\left(x_{i}\right)$ & $I_{2}^{\mathrm{Pr}}\left(x_{i}\right)$ \\
\hline \hline$x_{1}$ & 0.49677 & 0 & $x_{8}$ & 0 & 0.49942 \\
\hline$x_{2}$ & 0.49745 & 0 & $x_{9}$ & 0.49687 & 0.49760 \\
\hline$x_{3}$ & 0 & 0.49942 & $x_{10}$ & 0.49672 & 0 \\
\hline$x_{4}$ & 0.49686 & 0.49927 & $x_{12}$ & 0.49942 \\
\hline$x_{5}$ & 0.49672 & 0 & $x_{13}$ & 0.49681 & 0 \\
\hline$x_{6}$ & 0.49678 & 0 & & 0.49672 & 0 \\
\hline$x_{7}$ & 0.49752 & 0 & & \\
\hline
\end{tabular}

$I_{1}^{\mathrm{Pr}}\left(x_{4}=1\right)=P\left(T=1 \mid x_{4}=1\right)-P\left(T=1 \mid x_{4}=0\right)$

$=0.99372$

The state importance of node $x_{4} I_{1}^{\mathrm{Pr}}\left(x_{4}=1\right)$ when $\boldsymbol{T}=\mathbf{1}$ can be obtained by Eq. (13) as follows:

$I_{1}^{\mathrm{Pr}}\left(x_{4}\right)=\frac{1}{2}\left(I_{1}^{\mathrm{Pr}}\left(x_{4}=1\right)+I_{1}^{\mathrm{Pr}}\left(x_{4}=2\right)\right)$

$=\frac{1}{2} \sum_{v_{i}=1}^{2}\left[P\left(T=1 \mid x_{4}=v_{i}\right)-P\left(T=1 \mid x_{4}=0\right)\right]$

$=0.49686$

The state importance and the probability importance of other nodes can be obtained as shown in Table $\mathbf{3}$ and Table 4.

3) The posterior probability calculation.

Taking the node $x_{4}$ for example, the posterior probability of node $x_{4}$ can be obtained using Eq. (10) when system fault state $\boldsymbol{T}=\mathbf{1}$ :
$P\left(x_{4}=1 \mid T=1\right)=\frac{P\left(x_{i}=1, T=1\right)}{P(T=1)}$

$=\frac{\sum_{x_{1}, x_{2}, \cdots, x_{13}, \mathrm{y}_{1}, \mathrm{y}_{2}, \cdots, \mathrm{y}_{5}} P\left(x_{1}, x_{2}, \cdots, x_{13}, \mathrm{y}_{1}, \mathrm{y}_{2}, \cdots, \mathrm{y}_{5}, T=1, x_{4}=1\right)}{P(T=1)}$

$=53.0$

4) Reliability analysis.

The weaknesses of the system can be identified through the importance of the root node, and by increasing the reliability of links the system reliability can also be improved.

Table 3 shows that the effect of the current fault status of the root node on the system is as follows: in the state of slight fault, the node $x_{7}$ has the greatest impact; in the state of serious fault, the nodes $x_{3}, x_{8}, x_{11}$ have the greatest impact.

Table 4 shows that the effect of the root node on the system is as follows: in the state of slight fault, the node $x_{7}$ has the greatest impact; in the state of serious fault, the nodes $x_{3}$, $x_{8}, x_{11}$ have the greatest impact. 
Table 5. Priori \& Posterior Probability of Nodes $\left(10^{-3} / \mathrm{h}\right)$.

\begin{tabular}{|c|c|c|c|c|c|c|c|}
\hline Node & $\begin{array}{c}\text { Priori } \\
\text { Probability }\end{array}$ & $\begin{array}{c}\text { Posterior } \\
\text { Probability } \\
T=1\end{array}$ & $\begin{array}{c}\text { Posterior } \\
\text { Probability } \\
\quad T=2\end{array}$ & Node & $\begin{array}{c}\text { Priori } \\
\text { Probability }\end{array}$ & $\begin{array}{c}\begin{array}{c}\text { Posterior } \\
\text { Probability } \\
T=1\end{array} \\
\text { T }\end{array}$ & $\begin{array}{c}\begin{array}{c}\text { Posterior } \\
\text { Probability } \\
T=2\end{array} \\
\text { T }\end{array}$ \\
\hline & $v_{i}=1, v_{i}=2$ & $v_{i}=1, v_{i}=2$ & $v_{i}=1, v_{i}=2$ & & $v_{i}=1, v_{i}=2$ & $v_{i}=1, v_{i}=2$ & $v_{i}=1, v_{i}=2$ \\
\hline$x_{1}$ & $0.232,0$ & $45.5,0$ & $0.232,0$ & $x_{8}$ & $0,0.44$ & 0,0 & $0,273.8$ \\
\hline$x_{2}$ & $1.61,0$ & $315.9,0$ & $1.61,0$ & $x_{9}$ & $0.292,0.144$ & $57.3,0$ & $0.26,89.6$ \\
\hline$x_{3}$ & $0,0.44$ & 0,0 & $0,273.8$ & $x_{10}$ & $0.126,0$ & $24.7,0$ & $0.126,0$ \\
\hline$x_{4}$ & $0.270,0.144$ & $53.0,0$ & $0.24,89.6$ & $x_{11}$ & $0,0.44$ & 0,0 & $0,273.8$ \\
\hline$x_{5}$ & $0.126,0$ & $24.7,0$ & $0.126,0$ & $x_{12}$ & $0.322,0$ & $63.2,0$ & $0.322,0$ \\
\hline$x_{6}$ & $0.252,0$ & $49.4,0$ & $0.252,0$ & $x_{13}$ & $0.126,0$ & $24.7,0$ & $0.126,0$ \\
\hline$x_{7}$ & $1.75,0$ & $343.4,0$ & $1.75,0$ & & & & \\
\hline
\end{tabular}

These nodes mentioned above are needed to be paid attention to and to be improved.

According to the posterior probability of each root node, fault probability of each node can be gained after a system fault, and thus fault diagnosis for system can be made, that is according to the posterior probability, the corresponding root nodes are detected top down.

Table 5 summarizes when the slight system fault occurs, the order of checking slight fault nodes is $x_{7}, x_{2}, x_{12}, x_{9}, x_{4}, x_{6}$, $x_{1},\left(x_{5}, x_{10}, x_{13}\right)$; when serious system fault occurs, the order of checking serious fault nodes is $\left(x_{5}, x_{10}, x_{13}\right), x_{4}, x_{9}$, the order of checking slight fault nodes is $x_{7}, x_{2}, x_{12}, x_{9}, x_{6}, x_{4}, x_{1}$, $\left(x_{5}, x_{10}, x_{13}\right)$.

\section{CONCLUSION}

The proposed method combines the advantages of fuzzy set theory and BN technology to solve the problem of the uncertainty and fuzziness in reliability analysis of complex system.

In this paper, the triangular fuzzy number is adopted to describe the fuzzy probability of the event, which solves the problem of being difficult to obtain precise data; through the method of group decision, using DUOWA operator, the assessments of experts with uncertain weights are integrated, which solves the problem of the influence of uncertain weights to the results of assessment.

Bayesian network model is used to handle the uncertainty of logical relationship between events and to compute system reliability. Through the calculation of importance of node and posterior probabilities, a more effective decision support is offered for the design and maintenance of complex systems.

The analysis of cases has proved that this method provides a powerful analytical tool for the reliability analysis of complex systems in a complex environment.

\section{CONFLICT OF INTEREST}

The authors confirm that this article content has no conflict of interest.

\section{ACKNOWLEDGEMENTS}

This research was partially supported by the National Nature Science Foundation of China (No. 61203302).

\section{REFERENCES}

[1] S. P. Wang, Engineering reliability, Beihang University Pess: Beijing, 2000.

[2] L. D. Xing, and G. Levitin, "BDD-based reliability evaluation of phased-mission systems with internal/external common-cause failure", Reliability Engineering and System Safety, vol. 84, no. 2, pp. 145-153, 2013.

[3] R. X. Duan, J. L. Tu, and D. C. Dong, "A novel hybrid approach of fault tree and Bayesian networks for fault diagnosis", Journal of Computational Information System, vol. 6, no. 11, pp. 3605-3612, 2010.

[4] H. Langseth, and L. Portinale, "Bayesian networks in reliability", Reliability Engineering System Safety, vol. 92, no.1, pp. 92-108, 2007.

[5] Y. Jiang, H. Zhang, X. Y. Song, X. Jiao, H. N. N. Hung, M. Gu, and J. Sun, "Bayesian-network-based reliability analysis of plc systems", IEEE transactions on industrial electronics, vol. 60, no. 11, pp. 5325-5336, 2013.

[6] B. P. Cai, Y. Liu, Z. Liu, X. Tian, X. Dong, and S. Yu, "Using Bayesian networks in reliability evaluation for subsea blowout preventer control system", Reliability Engineering and System Safety, vol. 108, no. 2, pp. 32-41, 2012.

[7] K. Nima, K. Faisal, and A. Paul, "Safety analysis in process facilities: Comparison of fault tree and Bayesian network approaches", Reliability Engineering System Safety, vol. 96, no. 8, pp. 925-932, 2011.

[8] M. R. Martins, and M. C. Maturana, "Application of Bayesian Belief networks to the human reliability analysis of an oil tanker operation focusing on collision accidents", Reliability Engineering and System Safety, vol. 110, pp. 89-109, 2013.

[9] R. X. Duan, J. L. Tu, and D. C. Dong, "A novel hybrid approach of fault tree and Bayesian networks for fault diagnosis", Journal of Computational Information System, vol. 6, no.11, pp. 3605-3612, 2010.

[10] X. Y. Wang, and Y. H. Qiu, "Software dependability evaluation based on linguistic Bayesian network and OWA operator", Journal 
of Computational Information System, vol. 9, no. 7, pp. 2889-2896, 2013.

[11] S. Mahadevan, R. Zhang, and N. Smith, "Bayesian networks for system reliability reassessment", Structural Safety, vol. 23, pp. 231-251, 2001.

[12] A. G. Wilson, and A. V. Huzurbazar, "Bayesian networks for multilevel system reliability", Reliability Engineering and System Safety, vol. 92, no. 10, pp. 1413-1420, 2007.

[13] Y. Lu, Q. M. Li, and Z. P. Zhou, "Safety risk prediction of subway operation based on fuzzy Bayesian network", Chinese Journal of Southeast University, vol. 40, no. 5, pp. 1110-1114, 2010.
[14] C. C. Chang, R. S. Chen, and P. R. Sun, "Applying fuzzy Bayesian maximum entropy to extrapolating deterioration in repairable systems", (Y. Zhaf, ed.), Machine learning, Intech, 2010, pp. 217-238.

[15] C. Simon, and P. Weber, "Evidential networks for reliability analysis and performance evaluation of systems with imprecise knowledge", IEEE Transactions on Reliability, vol. 58, no.1, pp. 69-87, 2009.

[16] Z. S. XU, "Dependent uncertain ordered weighted aggregation operators", Information Fusion, vol. 9, no. 2, pp. 310-316, 2008.

Received: November 19, 2014

(C) Sheng et al.; Licensee Bentham Open.

This is an open access article licensed under the terms of the Creative Commons Attribution Non-Commercial License (http://creativecommons.org/licenses/by-nc/3.0/) which permits unrestricted, non-commercial use, distribution and reproduction in any medium, provided the work is properly cited. 